\title{
Development and validation of a mouse model of contemporary cannabis smoke exposure
}

\author{
Matthew F. Fantauzzi ${ }^{1,2}$, Steven P. Cass ${ }^{1,2}$, Joshua J.C. McGrath ${ }^{1,2}$, Danya Thayaparan ${ }^{1,2}$, Peiyao Wang ${ }^{2}$, \\ Martin R. Stampfli ${ }^{1,2,3}$ and Jeremy A. Hirota ${ }^{1,2,3}$
}

${ }^{1}$ Dept of Medicine, McMaster University, Hamilton, ON, Canada. ${ }^{2}$ McMaster Immunology Research Centre, McMaster University, Hamilton, ON, Canada. ${ }^{3}$ Firestone Institute for Respiratory Health - Division of Respirology, Dept of Medicine, McMaster University, Hamilton, ON, Canada.

Corresponding author: Jeremy A. Hirota (hirotaja@mcmaster.ca)

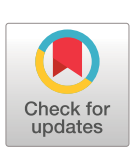

This version is distributed under the terms of the Creative Commons Attribution NonCommercial Licence 4.0. For commercial reproduction rights and permissions contact permissions@ersnet.org

This article has supplementary material available from openres.ersjournals.com

Received: 12 Feb 2021 Accepted: 5 May 2021

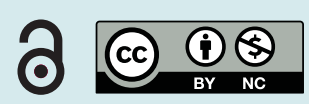

\author{
Shareable abstract (@ERSpublications) \\ Validation of the use of contemporary cannabis available on the legal market of known THC/CBD \\ composition in a mouse model of smoke exposure with readouts of lung inflammation \\ https://bit.ly/3okHWS4
}

Cite this article as: Fantauzzi MF, Cass SP, McGrath JJC, et al. Development and validation of a mouse model of contemporary cannabis smoke exposure. ERJ Open Res 2021; 7: 00107-2021

[DOI: 10.1183/23120541.00107-2021].

\section{Abstract}

Cannabis is widely used for both recreational and medicinal purposes. Inhalation of combusted cannabis smoke is the most common mode of drug consumption, exposing the lungs to the pharmacologically active ingredients, including tetrahydrocannabinol (THC) and cannabidiol (CBD). While the relationship between cannabis smoke exposure and compromised respiratory health has yet to be sufficiently defined, previous investigations suggest that cannabis smoke may dysregulate pulmonary immunity. Presently, there exist few preclinical animal models that have been extensively validated for contemporary cannabis smoke exposure.

To address this need, we developed a mouse model with readouts of total particulate matter, serum cannabinoid and carboxyhaemoglobin levels, lung cellular responses, and immune-mediator production.

Using a commercially available smoke exposure system and a cannabis source material of documented THC/CBD composition, we exposed mice to a mean \pm SD total particulate matter of $698.89 \pm 66.09 \mu \mathrm{g} \cdot \mathrm{L}^{-1}$ and demonstrate increases in serum cannabinoids and carboxyhaemoglobin. We demonstrate that cannabis smoke modulates immune cell populations and mediators in both male and female BALB/c mice. This modulation is highlighted by increases in airway and lung tissue macrophage populations, including tissueresident alveolar macrophages, monocyte-derived alveolar macrophages, and interstitial macrophage subpopulations. No changes in airway or lung tissue infiltration of neutrophils were observed. Immunemediator analysis indicated significant upregulation of macrophage-derived chemokine, thymus and activation-regulated chemokine, and vascular endothelial growth factor within the lung tissue of cannabis smoke-exposed mice.

This accessible and reproducible smoke-exposure model provides a foundation to explore the impact of chronic cannabis exposures and/or co-exposures with pathogens of clinical relevance, such as influenza.

\section{Introduction}

Cannabis is a mood-altering drug that is widely used for its psychoactive and remedial effects. It is the most consumed drug worldwide with an estimated 192 million users, a number that is expected to increase due to trends in recreational legalisation as well as innovations in medical applications [1]. The drug consists of over 400 biologically active compounds with $25 \%$ of these being cannabinoids, most notably tetrahydrocannabinol (THC) and cannabidiol (CBD) [2]. These compounds are thought to play roles in a variety of physiological processes, including neurocognition, appetite stimulation, pain alleviation, and immunity [3-6]. Notably, the method by which cannabis is consumed can both influence the pharmacological response to the drug and modulate the risk of collateral health effects. Among these routes, inhalation of combusted cannabis smoke is the most common mode of drug consumption, exposing 
the lungs to the pharmacologically active ingredients as well as a plethora of combustion products [7, 8]. Research investigating the potential health risks of inhaled smoke is highly warranted given our insufficient understanding of the biological consequences of cannabis smoke exposure.

Much like tobacco cigarette smoking, cannabis smoke exposure has been implicated in the development of adverse respiratory outcomes. Cannabis smoking is linked to respiratory symptomology, including an increased incidence of cough, wheezing, chest tightness, and shortness of breath [9-12]. Studies also demonstrate a potential link between cannabis use and an increased risk of pulmonary infection, suggesting that cannabis smoke may compromise respiratory host defence [13-16]. Although there is currently no strong association between cannabis smoking alone and the development of COPD, research indicates a potential synergistic effect between cannabis and tobacco smoke that may exacerbate lung inflammation and disease development $[17,18]$. While work delineating the exact mechanisms by which cannabis smoke promotes lung disease pathogenesis is limited, several studies have implicated dysregulated pulmonary immunity as a factor in these processes [19-21]. Despite this, limited experimental models are available to perform detailed assessments of how cannabis smoke exposure directly contributes to modulation of lung immunity.

While rodent models have been used in-depth to characterise tobacco smoke-associated lung inflammation, cannabis smoke studies using similar approaches remain largely unexplored. Historical in vivo investigations utilising cannabis smoke exposure systems require an update as the composition of cannabis has varied significantly over time and since legalisation [22, 23]. The average concentration of THC in a commercially available strain can exceed $15 \%$, whereas earlier models used strains with THC levels below $5 \%$. Consequently, early research on cannabis smoke may not accurately reflect the health implications of modern cannabis use. To date, few rodent smoke-exposure studies using compositionally relevant strains of cannabis exist [24], particularly those procured from legal suppliers. This omission, in conjunction with evolution in the commercial cannabis landscape, support the need for further investigations characterising the mechanisms through which cannabis smoke contributes to lung inflammation and pathology.

When considering how cannabis smoke exposure modulates immunological processes in the lungs, one can consider the well-characterised effects of tobacco cigarette smoke on pulmonary inflammation. Supporting this comparison are the chemical compositional similarities between the smoke of each combusted plant product, which suggests that any inflammatory effects may be conserved [8]. Studies investigating tobacco smoke exposure have identified key changes to immune cell population dynamics and function. Altered innate immunity has been suggested to be an early marker and inducer of smoke-associated disruption to lung homeostasis. In particular, it has been demonstrated that lung macrophages and neutrophils perform an essential role in mediating tobacco smoke-induced inflammation [25]. The central paradigm suggests that proinflammatory changes to lung macrophage function and quantity leads to pulmonary neutrophilia and subsequent exacerbated inflammation following tobacco smoke exposure [26, 27]. Previously, this paradigm centred around the role of tissue-resident alveolar macrophages, however, unexplored until recently macrophage subtypes such as monocyte-derived alveolar macrophages and interstitial macrophage (IM) populations 1-3 have been hypothesised to also play a role $[28,29]$. While these populations have yet to be shown to be involved in tobacco smoke-associated immunomodulation, their suggested contribution to proinflammatory outcomes in the lung supports their inclusion as possible effector cells in smoke-induced inflammation [29, 30]. The impact of cannabis smoke exposure on these macrophage populations and other immune cells potentially important in smoke-associated lung inflammation remains to be extensively unexplored.

In the present study, we develop a mouse model of contemporary cannabis smoke exposure using a legal source of defined composition. We confirm that our exposure system delivers cannabis smoke to mice using readouts of serum cannabinoids and carboxyhaemoglobin (COHb). Using a 4-day smoke exposure model, we saw the induction of changes to lung immune cellularity and mediator levels, suggesting that cannabis smoke is not innocuous. Importantly, we establish and validate the system using commercially available cannabis strains, devoid of potential confounding contaminants, which are consistent with relevant compositions available legally worldwide. 
Research Ethics Board of McMaster University. Number of animals per experiment are described in the figure legends with at least three animals per group $(n=3-10)$.

\section{Cannabis cigarette preparation}

Cannabis cigarettes were prepared using cannabis purchased from the Ontario Cannabis Store (Ontario, Canada). All strains used were indica dominant and contained 10-14\% THC and 0-2\% CBD. Cigarettes were hand-rolled by grinding dried cannabis flower, packing into king-size premier cigarette tubes (RJ Reynolds Tobacco Company, USA), and twisting off the end to seal the cigarette (figure 1a, b). Each cigarette contained $0.84 \pm 0.06 \mathrm{~g}$ of cannabis. Prior to running the smoke exposure protocol, the filter of each cigarette was removed, and the cannabis was packed towards the closed end (figure 1c).

\section{Cannabis smoke exposure protocol}

Prior to cannabis smoke exposure, mice were acclimatised to restrainers over 3 days. Mice were whole-body-exposed using a Smoke Inhalation Unit 24 (Promech, Sweden) (figure 1d) to the mainstream smoke of six cannabis cigarettes twice daily for 1 day or 4 days, depending on the experimental design. A single day exposure was used for a pharmacokinetic study. A 4-day exposure was used for a pharmacodynamic study with focus on lung immune profiling. Six cigarettes was chosen as an exposure amount based on efforts to model similar pharmacokinetic profiles between our mouse model and human exposures [31]. Throughout exposure, an exhaust system continuously pumps out excess air and smoke to maintain consistent chamber conditions. The entire system is located inside a fume hood with sidestream smoke from the burning end of the cigarette drawn away by the fume hood ventilation system. Additionally, the entire system is cleaned routinely to prevent the build-up of smoke residue to avoid third-hand exposure. The design of this system and our protocol isolates mainstream smoke exposure and minimises second- and third-hand smoke exposures. Mice were given a 3-h break in between daily exposure sessions. Control mice were sham-exposed to room air.

\section{Total particulate matter measurement}

Total particulate matter (TPM) was measured in the smoke exposure system using a Flowmeter 4045 (TSI Incorporated, USA) and paper filters. Six cigarettes were placed in the exposure system and burned using the same settings as the experimental smoke exposure procedure. Smoke and air outflow was measured during the burning of the first cigarette using the Flowmeter 4045. Filters were placed and subsequently removed one at a time in the outflow tubing during the burning of cigarettes one, three and five. Following

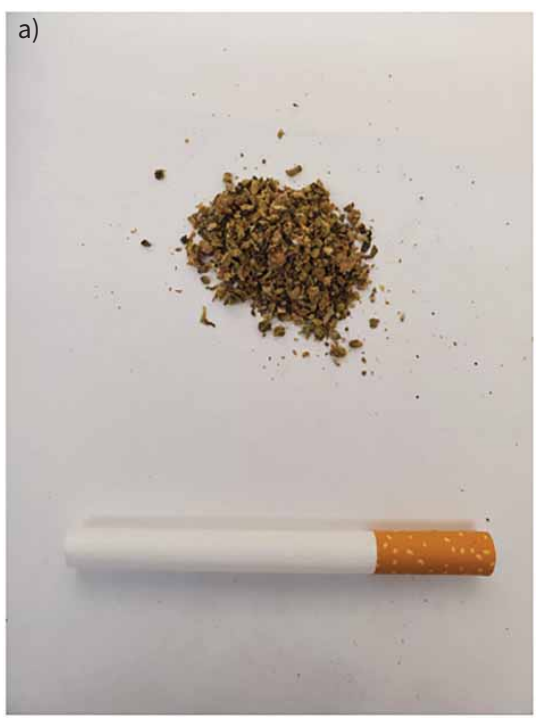

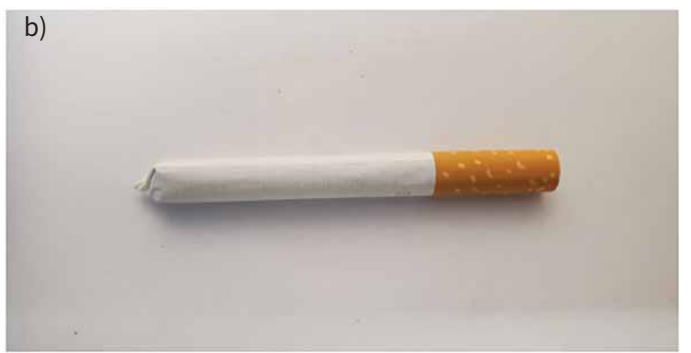

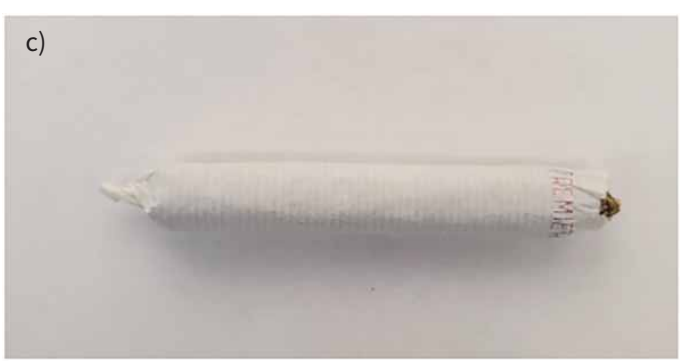

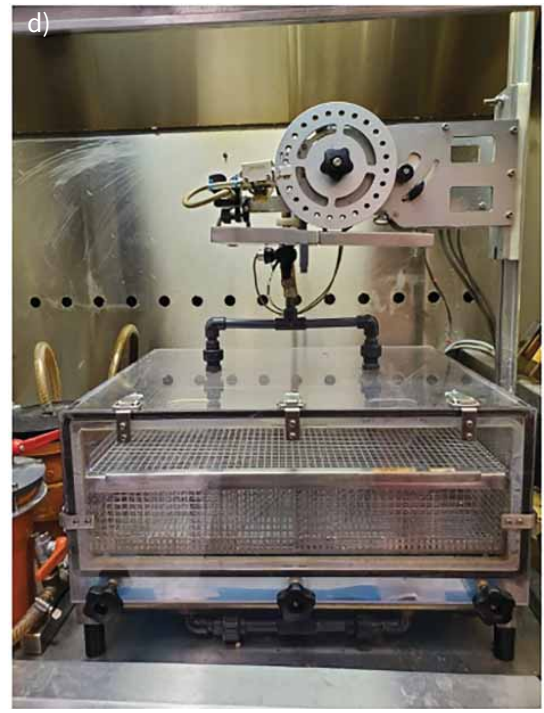

FIGURE 1 Cannabis cigarette preparation and smoke exposure system. Cannabis cigarettes are prepared by a) grinding $0.84 \pm 0.06 \mathrm{~g}$ dried cannabis flower (10-14\% tetrahydrocannabinol and $0 \%$ cannabidiol), b) packing into king-size empty cigarette tubes and twisting off the end to seal the cigarette. c) The filter of each cigarette is removed, and cannabis is packed towards the sealed end of the cigarette prior to smoke exposure. d) Cannabis cigarettes are loaded into the cigarette holder on a Smoke Inhalation Unit 24 system. Experimental animals are placed in the lower chamber. Cannabis smoke and fresh air are pumped into the chamber following a computerised protocol attached to the system. 
the exposure, the filters were weighed and TPM was derived by dividing the average change in filter mass by the average change in outflow multiplied by four.

\section{Carboxyhaemoglobin and cannabinoid measurement}

$15 \mathrm{~min}$ or $1 \mathrm{~h}$ following a smoke exposure period, whole blood was collected from smoke-exposed and control mice using capillary tubes via retro-orbital bleed. Heparinised capillary tubes were used to collect whole blood from the opposite eye. $\mathrm{COHb}$ in whole blood samples was quantified via CO-oximetry by the McMaster University Medical Centre core laboratory. Plasma was collected by incubating and centrifuging heparinised whole blood samples. Samples were prepared for THC, carboxy-THC, and CBD quantification via mass spectrometry by the McMaster University Centre for Microbial Chemical Biology. Briefly, samples were treated with cold acetonitrile containing $1 \%(\mathrm{v} / \mathrm{v})$ formic acid and $50 \mathrm{ng} \cdot \mathrm{mL}^{-1}$ of internal standard mixture. Next, high-performance liquid chromotography (HPLC)-grade water was added to the samples, which were then injected into an Agilent 1290 Infinity II HPLC coupled to an Agilent 6495C iFunnel QQQ mass spectrometer (Agilent, Santa Clara, CA, USA). Separated analytes were eluted and introduced to the mass spectrometer. Quantitation of each cannabinoid was based on the peak area measurement measured by the mass spectrometer. Pooled blank mouse plasma to be used as matrix background was processed using the same protocol as the samples. Standards of THC, carboxy-THC, and CBD were purchased (Sigma Aldrich Canada, Ontario, Canada), prepared in the matrix background, and processed using the same protocol as the samples.

\section{Bronchoalveolar lavage collection and cell differential quantification}

Following 4 days of cannabis smoke exposure, mice were anesthetised with isoflurane and euthanised via asphyxiation and cardiac puncture. Whole lungs and trachea were excised from the chest cavity. Bronchoalveolar lavage (BAL) was collected by cannulating the trachea, tying off the right lung lobe, instilling the left lobe with $250 \mu \mathrm{L}$ of cold sterile PBS, and re-aspirating the fluid. Two sequential instillations were performed in this manner and combined. BAL samples were centrifuged, supernatant removed, and resuspended in $130 \mu \mathrm{L}$ of PBS. Cell counts were performed on the resuspended samples via haemocytometer and light microscopy. Remaining samples were used for cell differentials via cytospins. BAL samples were centrifuged onto pre-wet microscope slides and then stained using Hema-3 fixative and solutions. Mononuclear and polymorphonuclear cell populations were identified and imaged using Zen microscopy imaging software (Zeiss International, Germany). Cell differentials were multiplied by cell counts to derive differential cell counts.

\section{Lung tissue processing and flow cytometric analysis}

Following instillation of the left lobe for BAL collection, the right lung lobe was excised and collected in RPMI. Collected lobes were incubated for $1 \mathrm{~h}$ at $37^{\circ} \mathrm{C}$ in $10 \mathrm{~mL}$ of cRPMI with 1500 units of 260 units $\cdot \mathrm{mg}^{-1}$ type 1 collagenase (cat. no. LS004194; Worthington Biochemical, Lakewood, NJ, USA) per lobe and pressed through a $35-\mu \mathrm{m}$ filter. Samples were centrifuged and treated with ammonium-chloride-potassium (ACK) lysis buffer. Following centrifugation, samples were resuspended in $1 \%$ bovine serum albumin with $2 \mathrm{mM}$ EDTA. Aliquots of the single-cell suspension were used for cell counts as outlined in the previous methods section. Single-cell suspensions were plated, centrifuged, treated with blocking solution, and stained with fluorophore-labelled antibodies. Samples were then assessed for immune cell population composition and quantity using BD LSRFortessa Flow Cytometry (BD Biosciences, Canada). The following antibodies were used for flow cytometric analysis (figure S1): LIVE/DEAD ${ }^{\mathrm{TM}}$ Fixable Yellow Dead Cell Stain kit (Thermo Fisher Scientific, cat. no. L34967), CD45-Alexa Fluor 700 (BioLegend, cat. no. 103127), B220-APC-Cy7 (BioLegend, cat. no. 103211), CD3e-APC-Cy7 (BioLegend, cat. no. 100329), EpCAM-APC-Cy7 (BioLegend, cat. no. 118217), Ly6G-BV785 (BioLegend, cat. no. 127645), CD11b-PE-Dazzle 594 (BioLegend, cat. no. 101255), CD64-PE-Cy7 (BioLegend, cat. no. 139313), MerTK-APC (BioLegend, cat. no. 151507), SiglecF-PE (BioLegend, cat. no. 155505), CD11c-BV650 (BioLegend, cat. no. 117339), CD24-BV421 (BioLegend, cat. no. 101825), MHCII-PerCP-Cy5.5 (BioLegend, cat. no. 107625), and Ly6C-BV510 (BioLegend, cat. no. 128033).

\section{Immune-mediator quantification}

Left lung lobes were collected following BAL instillation. Lobes were homogenised in PBS, centrifuged, and supernatant was collected. Immune mediators were quantified in lung tissue homogenate supernatant using the Mouse Cytokine Array/Chemokine Array 44-plex (Eve Technologies, Calgary, AB, Canada). Point-to-point semi-logarithmic analysis was applied to all immune meditator quantities. 
Statistical analysis

GraphPad Prism 9 (GraphPad Software, San Diego, CA, USA) was used for statistical analyses. The data were expressed in terms of mean and SEM. Unpaired t-tests were performed to compare the means of two groups within the same sex. Differences were considered statistically significant when $\mathrm{p}<0.05$.

Results

Cannabis smoke combustion, inhalation, and systemic distribution

We first sought to establish a mouse model of cannabis smoke exposure using a Smoke Inhalation Unit 24, which has previously been used in tobacco smoke-exposure studies (figure 1). Six cannabis cigarettes were selected for each exposure session based on preliminary experiments that qualitatively assessed animal behaviour and tolerance (data not shown). Combustion was quantified by TPM and carried out in a single smoke-exposure session. During exposure, the mean \pm SD TPM concentration within the exposure chamber was $698.89 \pm 66.09 \mu \mathrm{g} \cdot \mathrm{L}^{-1}$. Cannabis smoke-exposed mice had reduced levels of activity and ventilation rates (data not shown). Upon removal from the chamber, mice appeared lethargic and hunched but returned to normal activity levels and posture within $1 \mathrm{~h}$. Despite these behavioural changes, cannabis smoke exposure was well tolerated with no reduction in clinical score (data not shown). For comparative purposes, these changes in behaviour are also observed in our well-characterised tobacco cigarette smoke models using the same exposure system $[25,26]$.

Following removal from the smoke exposure chamber, blood was collected within 15 min for quantification of metabolites of combustion and phytocannabinoids. $\mathrm{COHb}$, a metabolite produced when haemoglobin is bound by carbon monoxide inhaled from combustion, was significantly elevated in both male and female smoke-exposed mice (figure 2a). The elevation in $\mathrm{COHb}$ returned to baseline levels $60 \mathrm{~min}$ after exposure in both males and females (figure S2a). To validate phytocannabinoid delivery through smoke exposure, plasma samples were analysed via mass spectrometry (figure 2b). Plasma THC and carboxy-THC were both present in all male and female smoke-exposed mice compared to controls, which had no traces of either cannabinoid detected. CBD was not detected in any tested samples, consistent with the composition of the selected cannabis strains (reported as $10-14 \%$ THC and $0-2 \%$ CBD). In plasma samples collected 60 min after exposure, THC was detected in one of five males and one of five females, whereas carboxy-THC was detected in three of five males and three of five females (figure S2b). Plasma samples with detectable THC and carboxy-THC had levels only slightly above the lower level of detection for the analysis. Similar to the earlier assessment, CBD was undetected in all samples at $60 \mathrm{~min}$ after exposure.

Collectively, our results demonstrated that a commercially available smoke exposure system is able to combust cannabis material filled into preformed cigarette tubes, resulting in qualitative behaviour changes and systemic cannabinoid distribution consistent with expected metabolite kinetics.

\section{Cannabis smoke exposure modulates immune cell populations in the airways}

Next, we characterised the effects of cannabis smoke exposure on respiratory immune cell populations and immune mediators using a 4-day model. Previous investigations from our laboratory demonstrated that 4 days of smoke exposure is the minimum required to observe dysregulation in lung immunity [32, 33]. Total immune cellularity was significantly increased in female cannabis smoke-exposed BAL samples compared to controls (figure 3). Differential cell analysis demonstrated that this increase was driven by an expansion of mononuclear cells; no polymorphonuclear cells were present in any of the female samples. Total immune cellularity and mononuclear cell quantities did not significantly change in male cannabis smoke-exposed BAL samples.

Cannabis smoke exposure modulates immune cell populations in the lungs

Flow cytometric analysis of lung tissue samples further demonstrated modulation of immune cell populations with some distinct differences between sexes (figure 4). The proportion of total CD $45^{+}$cells in the lungs remained unchanged in cannabis-exposed animals, regardless of sex. However, in female cannabis-exposed mice, the proportion of eosinophils were significantly increased; total macrophages, neutrophils, and monocytes were unchanged; and dendritic cells were significantly decreased. In male cannabis-exposed mice, the proportion of macrophages were significantly increased; neutrophils, dendritic cells, and eosinophils were unchanged; and monocytes were significantly decreased.

Specific lung macrophage subpopulations have been suggested to be important in the development of lung inflammation [28, 30]. We therefore included complementary cell markers in our flow cytometric analysis to identify relevant subtypes (figure 5). In female cannabis-exposed mice, only the proportion of IM1s were significantly increased; all other subpopulations were unchanged. In male cannabis-exposed mice, 

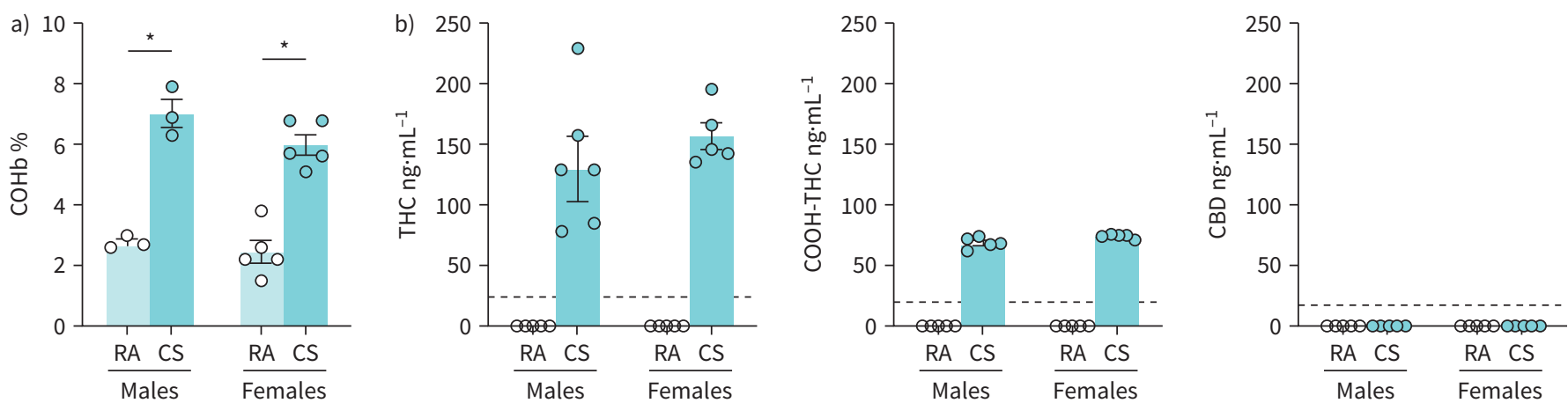

FIGURE 2 Acute cannabis smoke exposure increases plasma carboxyhaemoglobin (COHb), tetrahydrocannabinol (THC) and carboxy-THC $(\mathrm{COOH}-\mathrm{THC})$. Male and female 6-8-week-old BALB/c mice were exposed to room air (RA) or the smoke of six cannabis cigarettes (CS) twice in a day. Whole blood was collected via retro-orbital bleed within $15 \mathrm{~min}$ following the second exposure session. a) $\mathrm{COHb}$ percentage was quantified via CO oximetry. b) Plasma THC, COOH-THC and cannabidiol (CBD) were quantified via mass spectrometry. Dotted lines represent the limit of detection for specific cannabinoids. Data points at $0 \mathrm{ng} \cdot \mathrm{mL}^{-1}$ represent values below the limit of detection in cannabinoid analysis. Data represent mean \pm SEM; $n=3-5$ per group. ${ }^{*} \mathrm{p}<0.05$, unpaired t-test within each sex in $\mathrm{COHb}$ analysis.

tissue-resident alveolar macrophages, monocyte-derived alveolar macrophages, and IM1s were significantly increased, whereas IM2s and IM3s were unchanged as a proportion of total leukocytes, compared to control. While most subpopulations were detected in all samples, population size as a proportion of total leukocytes was near zero for some samples, notably within the monocyte-derived alveolar macrophage and IM3 subpopulations.

Overall, our results demonstrate that a 4-day cannabis smoke-exposure protocol results in modest changes in immune cell populations within the airways and lungs. Notably, while trends are largely conserved between female and males, lung macrophage populations are differentially affected by cannabis smoke exposure.

Impact of cannabis smoke exposure on immune mediators in the lungs

To perform a broad survey of the impact of cannabis smoke exposure on lung immune responses, we performed a 44-plex cytokine array on homogenised lung tissue (for all data see table S1). Interleukin (IL)- $1 \alpha$, an IL-1 family cytokine that has been demonstrated to be involved in smoke-associated inflammation [27], was unchanged in cannabis smoke-exposed mice compared to controls (figure 6a).
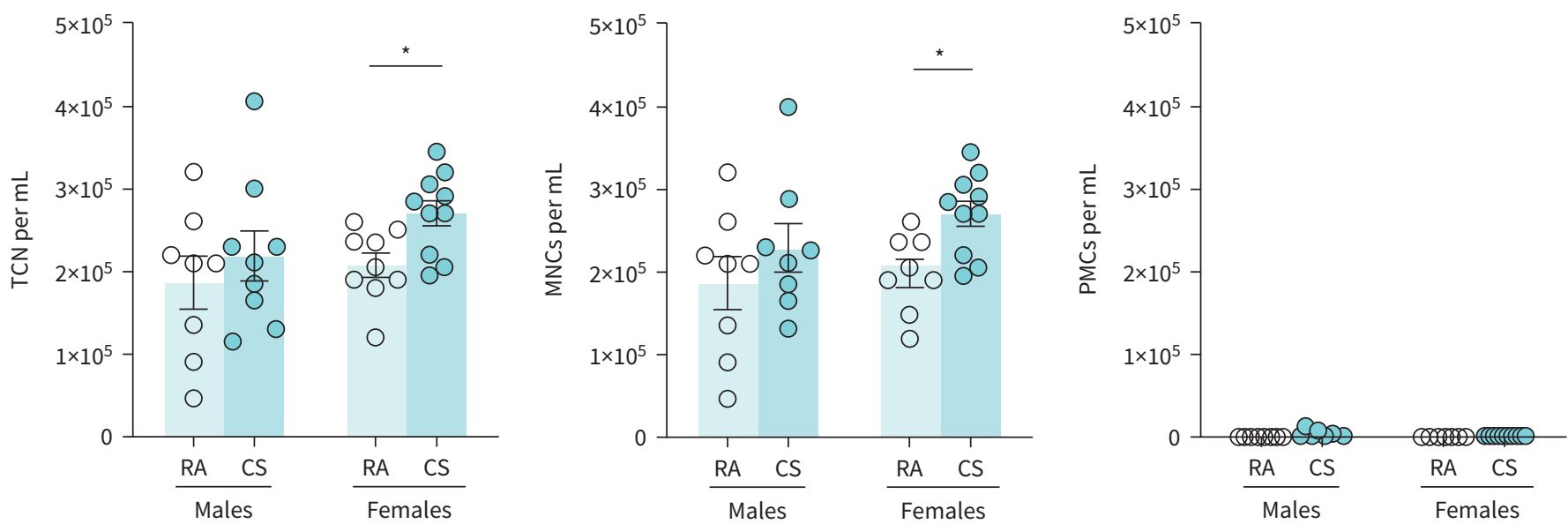

FIGURE 3 Cannabis smoke exposure modulates cellularity in the bronchoalveolar lavage (BAL). Male and female 6-8-week-old BALB/c mice were exposed to room air (RA) or the smoke of six cannabis cigarettes (CS) twice a day for 4 days. a) BAL total cell number (TCN), mononuclear cells (MNCS), and polymorphonuclear cells (PMCS) were determined via haemocytometer and cytospin differentials. Data represent mean \pm SEM; $\mathrm{n}=8-10$ per group, data pooled from two identical experiments. ${ }^{*} \mathrm{p}<0.05$, unpaired $\mathrm{t}$-test within each sex. 

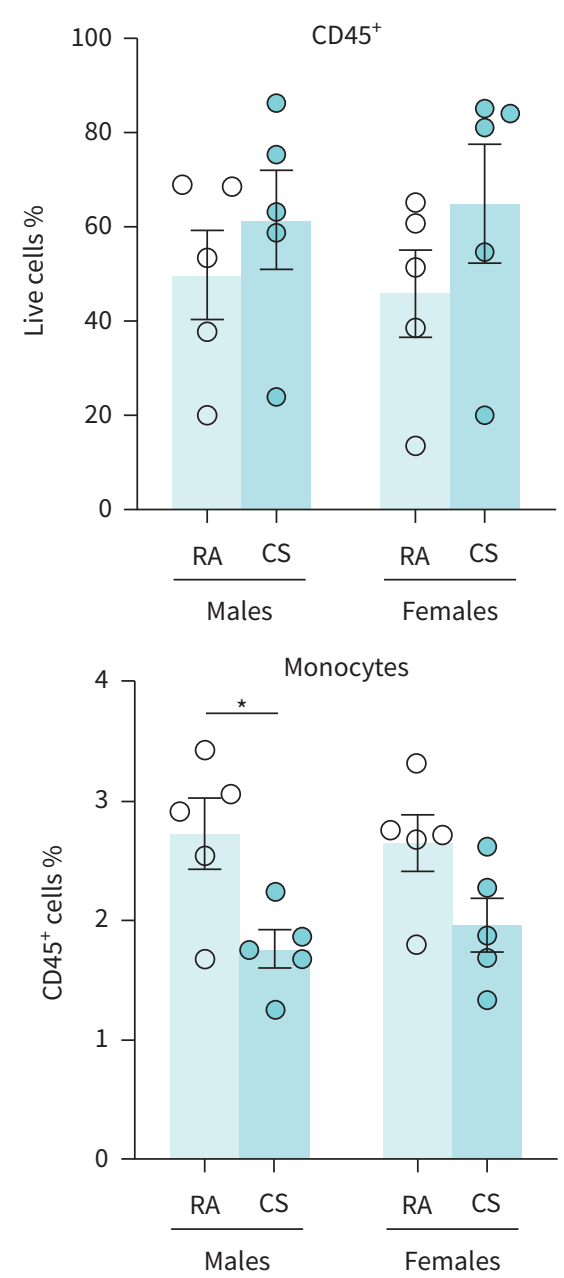
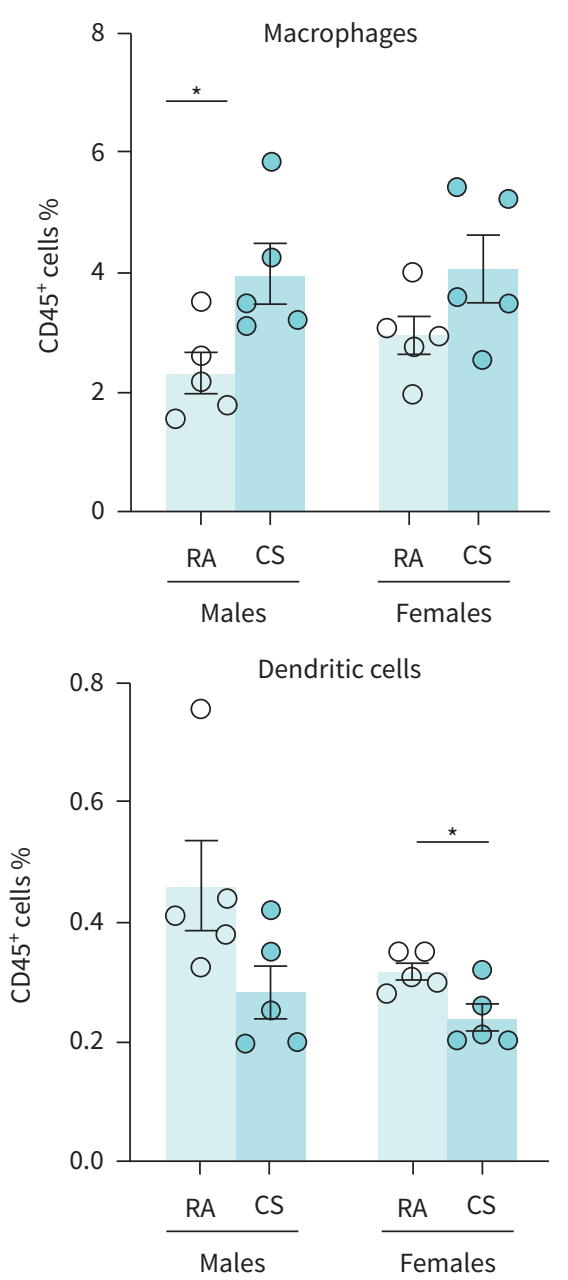
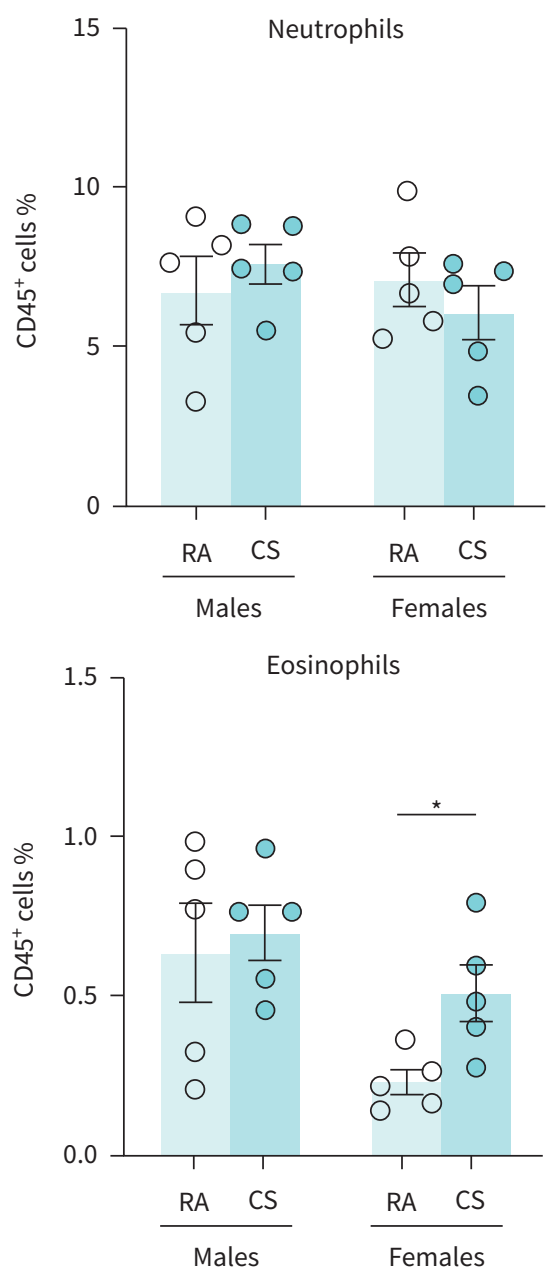

FIGURE 4 Cannabis smoke exposure modulates the proportionality of innate immune cell populations in the lungs. Male and female 6-8-week-old $\mathrm{BALB} / \mathrm{c}$ mice were exposed to room air (RA) or the smoke of six cannabis cigarettes (CS) twice a day for 4 days. Lung innate immune cell populations were quantified via flow cytometry. Proportionality was determined via haemocytometer. Data represent mean \pm SEM; $\mathrm{n}=5 \mathrm{per}$ group. ${ }^{*} p<0.05$, unpaired t-test within each sex.

Tissue-resident AMs

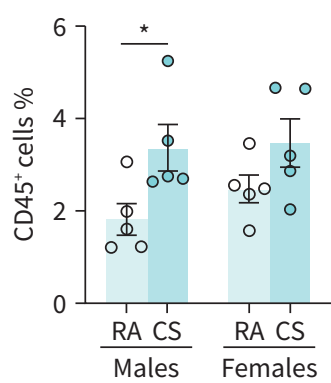

Monocyte-derived AMs

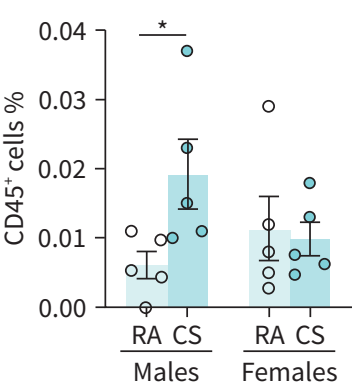

IM1

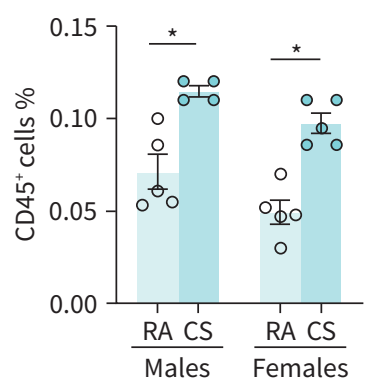

IM2

IM3

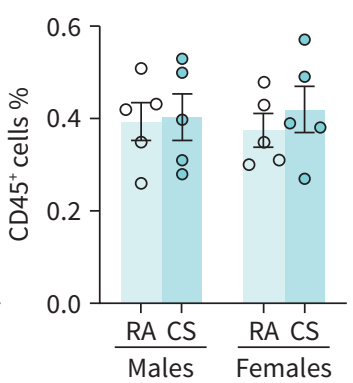

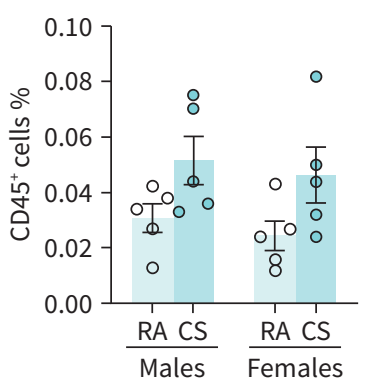

FIGURE 5 Cannabis smoke exposure modulates the proportionality of macrophage subpopulations in the lungs. Male and female 6-8-week-old BALB/c mice were exposed to room air (RA) or the smoke of six cannabis cigarettes (CS) twice a day for 4 days. Macrophage subpopulations were quantified via flow cytometry. Proportionality was determined via haemocytometer. AM: alveolar macrophage; IM: interstitial macrophage. Data represent mean \pm SEM; $n=4-5$ per group. ${ }^{*} p<0.05$, unpaired $t$-test within each sex. 

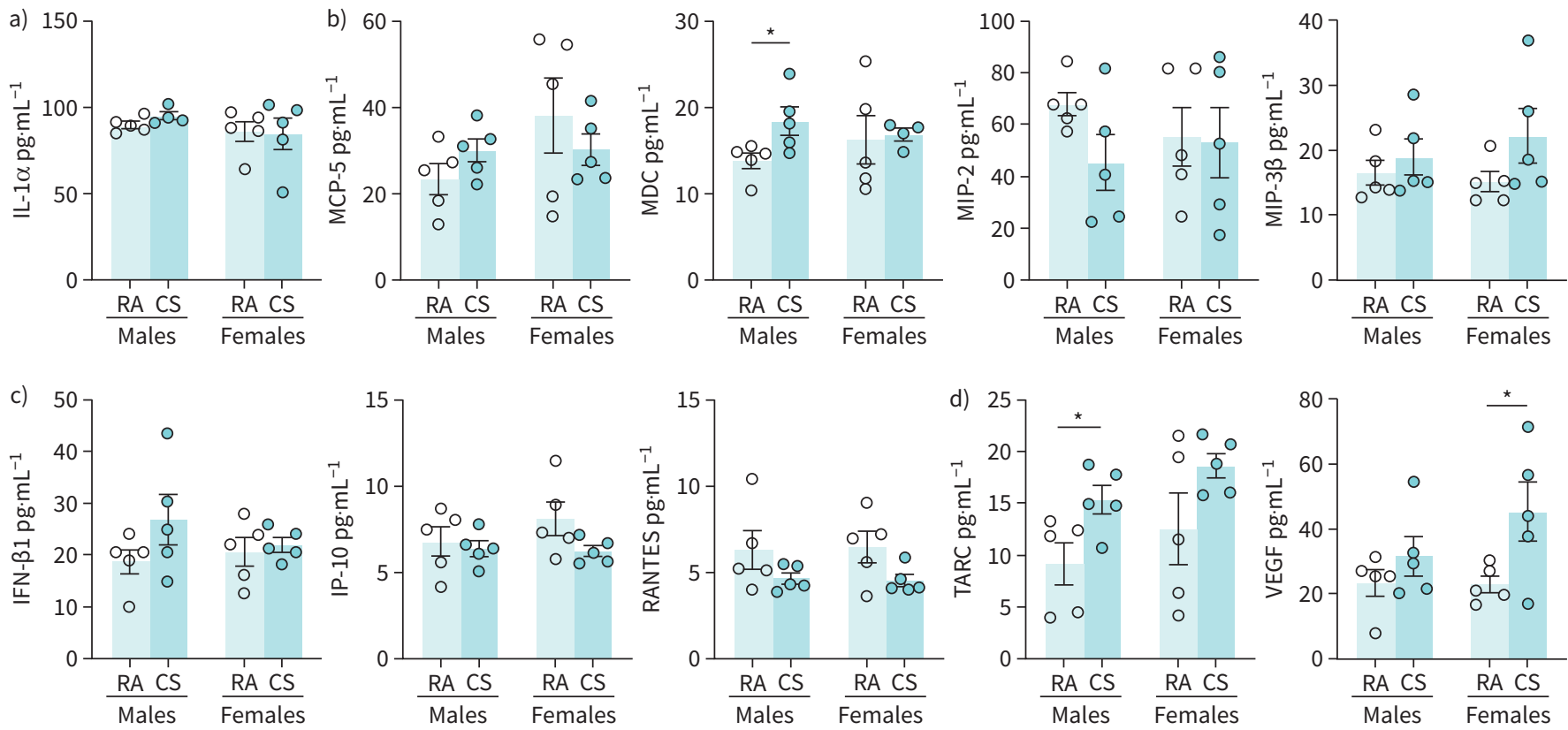

FIGURE 6 Impact of acute cannabis smoke exposure on immune mediators in the lungs. Male and female 6-8-week-old BALB/c mice were exposed to room air (RA) or the smoke of six cannabis cigarettes (CS) twice a day for 4 days. Immune mediators were quantified via multiplex analysis (Eve Technologies). Mediators shown are those with quantities above the lower level of detection of the analysis and are associated with a) smoke-associated inflammation, b) macrophage cell signalling, and c) antiviral signalling. d) Other immune mediators with significant differences above the lower level of detection are also shown. IL: interleukin; MCP: monocyte chemoattractant protein; MDC: macrophage-derived chemokine; MIP: macrophage inflammatory protein; IFN: interferon; IP-10: interferon- $\gamma$-inducible protein 10; TARC: thymus and activation-regulated chemokine; VEGF: vascular endothelial growth factor. Data represent mean \pm SEM; $n=5$ per group. ${ }^{*} p<0.05$, unpaired t-test within each sex.

Detectable macrophage-associated immune mediators such as MCP-5, MIP-2, and MIP-3 $\beta$ were also unchanged, whereas macrophage-derived chemokine (MDC) was significantly increased in male smoke-exposed mice (figure 6b). Antiviral cytokines, which are typically involved in early innate immune signalling in response to viral infection and have been proposed to be suppressed by cannabis smoke exposure [34, 35], such as interferon (IFN)- $\beta-1$, IP-10 (CXCL10), and RANTES (CCL5), were unchanged in cannabis smoke-exposed mice compared to control (figure 6c). Additional cytokines including CCL17 (thymus and activation-regulated chemokine (TARC)) in the males $(\mathrm{p}=0.037)$ and vascular endothelial growth factor (VEGF) in the females were significantly increased compared to room air control (figure 6d), with trends conserved across both sexes.

In summary, immune profiling in lung tissue following cannabis smoke exposure reveals elevations in TARC and VEGF, with limited impact on IL-1 family cytokines, macrophage and monocyte chemotactic factors, and antiviral mediators.

\section{Discussion}

The increasing popularity of smoking cannabis recreationally and medicinally has brought to light the absence of experimental evidence of the potential lung health risks associated with inhalation. Given that our limited knowledge is based on historical data where cannabis compositions significantly varied, we sought to understand the effect of inhaled cannabis smoke using a well-controlled system and compositionally relevant cannabis strains [22, 23]. We demonstrated that cannabis smoke exposure resulted in systemic distribution of cannabinoids and associated behaviour changes. Lung immune profiling reveals consistent patterns between male and female mice, with changes in monocyte and macrophage cell populations and immune mediators. Our results form a modern foundation for further exploration of different strains of cannabis, exposure protocols, and interaction with pathogens important in lung health and disease.

The development of this cannabis smoke-exposure model was based heavily on existing models of tobacco smoke exposure that have been successfully used in respiratory investigations, among others [25]. To note, 
cannabis provides unique challenges in terms of model development compared to tobacco due to the variety of strain options and lack of controlled, research-grade cigarette products available. As such, we opted to establish our model using indica-dominant strains with THC levels of 10-14\% and CBD levels of $0-2 \%$ due to the popularity of this composition among recreational users [22]. At the time of this report, no licensed cannabis retailers in Canada manufacture identical cannabis cigarettes on a scale large enough to facilitate research. Thus, we developed an in-house method to produce consistent cigarettes using ground dried cannabis (figure 1). This in-house method is scalable to other research labs. In addition, we assessed both male and female mice, given the growing body of evidence that sex differences play an integral role in cannabis metabolism and cannabinoid signalling [36-38]. We suggest that future cannabis studies should aim to provide at minimum the strain name and THC and CBD concentrations to enable comparison between studies. Lot numbers and date of production could also allow for some between study comparisons. With these experimental variables implemented, our cannabis smoke exposure system effectively models real-world cannabis use patterns while respecting the need for reliability in a research environment.

Our cannabis smoke-exposure model was effective at delivering cannabis smoke to mice. Within the exposure chamber, mice displayed common symptoms of smoke exposure seen in our previous tobacco studies but tolerated smoke exposure well $[25,39]$. While studies have demonstrated that cannabis smoke increases locomotion in rats [40], our model appears to have the opposite effect, potentially due to the opposing effects of prolonged time in the smoke-filled exposure chamber. TPM in the chamber was seen to be at similar levels to those seen in tobacco smoke studies using the same system [25, 39], which may also contribute to the tobacco smoke-like behavioural phenotype we observed. While TPM alone contributes to lung inflammation [41, 42], cannabis smoke contains a myriad of other chemical components, cannabinoids, and reactive oxygen species, which can alter cellular and immunological functions. As such, any effects we observe cannot be attributed to TPM or the chemical composition of cannabis alone. Looking beyond TPM to quantify total carbon black, polycyclic aromatic hydrocarbons, and other aromatic amines reported to exist with tobacco and cannabis consumption $[7,8]$ should be considered for future studies to enable between study comparisons. In our post-exposure analysis, we found elevated levels of $\mathrm{COHb}$, THC, and carboxy-THC across the sexes that decreased $60 \mathrm{~min}$ after exposure (figure 2, figure S2). These increases, along with the lack of CBD present, are similar to pharmacokinetic trends in human cannabis smokers after cigarette consumption [31]. With these findings, we developed a model system, which resulted in the systemic delivery of smoke and cannabinoids at a magnitude equivalent with human smokers.

Maintaining immune homeostasis in the lungs is essential in supporting positive respiratory health outcomes. Previous studies have demonstrated a strong link between tobacco smoke exposure, chronic pulmonary inflammation, and the development of COPD [43], a relationship not yet fully explored in terms of cannabis smoke. Instigating this relationship is a smoke-induced modulation of immune cell populations and mediators leading to a chronic state of lung inflammation and tissue damage. In particular, innate immune cell populations such as lung macrophages and neutrophils have been found to be the main effector cells in the development of smoke-associated lung inflammation [25-27]. Given this paradigm, we initiated our characterisation of cannabis smoke-induced alterations to respiratory immunity by investigating whether cannabis smoke exposure alters immune cell populations. Our findings show that 4 days of cannabis smoke exposure modulated innate immune cell populations in the airways and lung tissue (figures 3-5). Specifically, we found that macrophages were increased in the airways of female smoke-exposed mice and the lung tissue of male smoke-exposed mice. These increases were matched by decreases in monocyte populations, suggesting that monocyte activation and differentiation may be induced as a consequence of cannabis smoke exposure. In addition, our analysis of lung macrophage subpopulations demonstrates a potential effect on subtypes that have been associated with heightened inflammatory profiles and may contribute to the development of lung pathologies [28, 30]. Conversely, our data show that 4 days of cannabis smoke exposure did not lead to any changes in neutrophils in the airways or lung tissue. While the current tobacco smoke literature suggests that exacerbated neutrophilia in the lungs is a prominent contributor to smoke-associated chronic inflammation, increases in macrophage quantity and phenotypical changes are thought to be the driver of exacerbated neutrophilic infiltration into smoke-exposed lung tissue [26]. Therefore, our findings are consistent with the hypothesis that acute cannabis smoke exposure may be inducing the early population level immunomodulation that characterises tobacco smoke-associated chronic inflammation.

Along with immune cell dysregulation, elevated proinflammatory cytokine levels have been associated with the induction of chronic lung inflammation and the development of COPD [44]. Tobacco and cannabis smoke has been shown to modulate immune mediators involved in antiviral signalling [34, 35]. 
Our immune-mediator analysis demonstrates a subtle modulation of the detected cytokines and chemokines in the lung tissue after 4 days of cannabis smoke exposure (figure 5). Of the detected mediators, our findings indicated increases of MDC, CCL17 (TARC), and VEGF in both sexes. MDC and TARC are both macrophage-associated mediators that have been previously demonstrated to be increased by tobacco smoke exposure [45]. Similarly, VEGF, which is highly expressed in the lung epithelium, has been shown to be greatly elevated by tobacco smoking [46]. Combined, these changes demonstrate further correlations between cannabis and tobacco smoke-induced effects on lung immunity. Conversely, mediators previously implicated in tobacco smoke investigations, such as IL-1 $\alpha$, were not altered. This suggests that cannabis smoke may have differential immunomodulator effects on the lung environment. As well, other macrophage and monocyte-associated mediators involved in activation and differentiation were undetected or unchanged. Antiviral signalling molecules such as IFN- $\beta-1$, IP-10, and RANTES were also unchanged. Consequently, the overall change to the inflammatory environment as a result of 4 days of cannabis smoke exposure was minimal but suggested that longer exposure protocols are warranted to understand chronic cannabis use.

Updated models of cannabis smoke exposure are needed to investigate how modern cannabis strains, which significantly vary from historical strains, impact lung health and disease. To address this unmet need, we characterised a model of smoke exposure, focusing on metabolic analysis for phytocannabinoids, and lung immune profiling, in both male and female mice. Our model system recapitulates the behavioural and pharmacokinetic changes observed in human cannabis smokers, providing evidence that the exposure protocol is within real-world ranges. Cannabis exposure resulted in innate immune cell changes that are proposed to precede more chronic inflammation in tobacco smokers using similar exposure systems. Our model lays a foundation for long-term exposures, which will add to our understanding of how immune cell populations, immune mediators, and lung structure are impacted by cannabis, and whether this contributes to adverse lung health outcomes. Similarly, our model can facilitate future investigations, which assess the relationship between cannabis smoke exposure and lung markers of COPD, providing insight into how cannabis smoke affects COPD pathogenesis. In addition, in both acute and chronic scenarios, our model can be leveraged for smoke/pathogen co-exposures to interrogate the potential pathological consequences of the cannabis smoke-induced immune changes observed. Collectively, our results define a validated modern cannabis smoke-exposure model essential for studying the relationship between cannabis consumption and respiratory health; an increasingly important undertaking given the growing popularity of cannabis on a global scale.

Acknowledgements: The authors acknowledge Joanna Kasinska (McMaster Immunology Research Centre) for her technical expertise and contributions to model development. Additionally, the authors acknowledge Tracey Campbell and Nikki Henriquez (McMaster University Centre for Microbial Chemical Biology) and Stephen Hill (McMaster University Medical Centre Core Laboratory) for their assistance in metabolite quantification. Lastly, Allen Fein (Michael G. De Groote Centre for Medicinal Cannabis Research) is acknowledged for his oversight and execution of our cannabis research license through Health Canada.

Conflict of interest: M.F. Fantauzzi has nothing to disclose. S.P. Cass has nothing to disclose. J.J.C. McGrath has nothing to disclose. D. Thayaparan has nothing to disclose. P. Wang has nothing to disclose. M.R. Stampfli reports an employment opportunity pursued at CSL Behring outside the submitted work. J.A. Hirota is a cofounder of Infinotype, a startup company in the bioinformatics space, outside the submitted work.

Support statement: This work was supported by the Ontario Lung Association and the Michael G. DeGroote Centre for Medicinal Cannabis Research. Funding information for this article has been deposited with the Crossref Funder Registry.

\section{References}

1 United Nations Office on Drugs and Crime (UNODC). World Drug Report 2018. Vienna, United Nations publication, 2018. www.unodc.org/wdr2018/

2 Health Canada. Information for Health Care Professionals: Cannabis (Marihuana, Marijuana) and the Cannabinoids. Ottawa, Health Canada, 2018.

3 Nader DA, Sanchez ZM. Effects of regular cannabis use on neurocognition, brain structure, and function: a systematic review of findings in adults. Am J Drug Alcohol Abuse 2018; 44: 4-18.

4 Jin LZ, Rangan A, Mehlsen J, et al. Association between use of cannabis in adolescence and weight change into midlife. PLOS ONE 2017; 12: e0168897. 
5 Baron EP. Medicinal properties of cannabinoids, terpenes, and flavonoids in cannabis, and benefits in migraine, headache, and pain: an update on current evidence and cannabis science. Headache 2018; 58: 1139-1186.

6 Costiniuk CT, Jenabian M-A. Cannabinoids and inflammation. AIDS 2019; 33: 2273-2288.

7 Novotný M, Merli F, Wiesler D, et al. Fractionation and capillary gas chromatographic-mass spectrometric characterization of the neutral components in marijuana and tobacco smoke condensates. $J$ Chromatogr $A$ 1982; 238: 141-150.

8 Moir D, Rickert WS, Levasseur G, et al. A comparison of mainstream and sidestream marijuana and tobacco cigarette smoke produced under two machine smoking conditions. Chem Res Toxicol 2008; 21: 494-502.

9 Tan WC, Bourbeau J, Aaron SD, et al. The effects of marijuana smoking on lung function in older people. Eur Respir J. 2019; 54: 1900826.

10 Gong H, Fligiel S, Tashkin DP, et al. Tracheobronchial changes in habitual, heavy smokers of marijuana with and without tobacco. Am Rev Respir Dis 1987; 136: 142-149.

11 Yayan J, Rasche K. Damaging effects of cannabis use on the Lungs. Adv Exp Med Biol 2016; 952: 31-34.

12 Tashkin DP. Marijuana and lung disease. Chest 2018; 154: 653-663.

13 Reiss CS. Cannabinoids and viral infections. Pharmaceuticals (Basel) 2010; 3: 1873-1886.

14 Ho T, Dasgupta A, Hargreave FE, et al. The use of cellular and molecular biomarkers to manage COPD exacerbations. Expert Rev Respir Med 2017; 11: 403-411.

15 Tetrault JM, Crothers K, Moore BA, et al. Effects of marijuana smoking on pulmonary function and respiratory complications: a systematic review. Arch Intern Med 2007; 167: 221-228.

16 Polen MR, Sidney S, Tekawa IS, et al. Health care use by frequent marijuana smokers who do not smoke tobacco. West J Med. 1993; 158: 596-601.

17 Morris MA, Jacobson SR, Kinney GL, et al. Marijuana use associations with pulmonary symptoms and function in tobacco smokers enrolled in the subpopulations and intermediate outcome measures in COPD Study (SPIROMICS). Chronic Obstr Pulm Dis 2018; 5: 46-56.

18 Tashkin DP. Does smoking marijuana increase the risk of chronic obstructive pulmonary disease? CMAJ 2009; 180: 797-798.

19 Bailey KL, Wyatt TA, Katafiasz DM, et al. Alcohol and cannabis use alter pulmonary innate immunity. Alcohol 2018; 80: 131-138.

20 Roth MD, Arora A, Barsky SH, et al. Airway inflammation in young marijuana and tobacco smokers. Am J Respir Crit Care Med 1998; 157: 928-937.

21 Ghasemiesfe M, Ravi D, Vali M, et al. Marijuana use, respiratory symptoms, and pulmonary function: a systematic review and meta-analysis. Ann Intern Med 2018; 169: 106-115.

22 Cascini F, Aiello C, Di Tanna G. Increasing delta-9-tetrahydrocannabinol ( $\delta$-9-THC) content in herbal cannabis over time: systematic review and meta-analysis. Curr Drug Abuse Rev 2012; 5: 32-40.

23 Smart R, Caulkins JP, Kilmer B, et al. Variation in cannabis potency and prices in a newly legal market: evidence from 30 million cannabis sales in Washington state. Addiction 2017; 112: 2167-2177.

24 Helyes Z, Kemény Á, Csekö K, et al. Marijuana smoke induces severe pulmonary hyperresponsiveness, inflammation, and emphysema in a predictive mouse model not via CB1 receptor activation. Am J Physiol Lung Cell Mol Physiol 2017; 313: L267-L277.

25 Botelho FM, Gaschler GJ, Kianpour S, et al. Innate immune processes are sufficient for driving cigarette smoke-induced inflammation in mice. Am J Respir Cell Mol Biol 2010; 42: 394-403.

26 Morissette MC, Shen P, Thayaparan D, et al. Disruption of pulmonary lipid homeostasis drives cigarette smoke-induced lung inflammation in mice. Eur Respir J 2015; 46: 1451-1460.

27 Botelho FM, Bauer CM, Finch D, et al. IL-1 $\alpha / \mathrm{IL}-1 \mathrm{R} 1$ expression in chronic obstructive pulmonary disease and mechanistic relevance to smoke-induced neutrophilia in mice. PLOS ONE 2011; 6: e28457.

28 Chakarov S, Lim HY, Tan L, et al. Two distinct interstitial macrophage populations coexist across tissues in specific subtissular niches. Science 2019; 363: eaau0964.

29 Murray PJ, Wynn TA. Protective and pathogenic functions of macrophage subsets. Nat Rev Immunol 2011; 11: 723-737.

30 McCubbrey AL, Barthel L, Mohning MP, et al. Deletion of c-FLIP from CD11bhi macrophages prevents development of bleomycin-induced lung fibrosis. Am J Respir Cell Mol Biol. 2018; 58: 66-78.

31 Huestis MA. Human cannabinoid pharmacokinetics. Chem Biodivers 2007; 4: 1770-1804.

32 Bauer CMT, Zavitz CCJ, Botelho FM, et al. Treating viral exacerbations of chronic obstructive pulmonary disease: insights from a mouse model of cigarette smoke and H1N1 influenza infection. PLOS ONE 2010; 5: e13251.

33 Morissette MC, Jobse BN, Thayaparan D, et al. Persistence of pulmonary tertiary lymphoid tissues and anti-nuclear antibodies following cessation of cigarette smoke exposure. Respir Res. 2014; 15: 49.

34 Huff RD, Aguiar JA, Tse W. Effect of long-acting $\beta$-agonists/glucocorticoids on human airway epithelial cell cytokine, transcriptomic and oxidative stress responses to cannabis smoke. ERJ Open Res 2020; 6 : 00265-2019. 
Chandiramohan A, Dabaghi M, Aguiar JA, et al. Development and validation of an open-source, disposable, 3D-printed in vitro environmental exposure system for Transwell ${ }^{\circledR}$ culture inserts. ERJ Open Res 2021; 7: 00705-02020.

36 Fattore L, Fratta W. How important are sex differences in cannabinoid action? Br J Pharmacol. 2010; 160: 544-548.

37 Fantauzzi MF, Aguiar JA, Tremblay BJ-M, et al. Expression of endocannabinoid system components in human airway epithelial cells - impact of sex and chronic respiratory disease status. ERJ Open Res 2020; 6: 00128-02020.

38 Nia AB, Mann C, Kaur H, et al. Cannabis use: neurobiological, behavioral, and sex/gender considerations. Curr Behav Neurosci Rep 2018; 5: 271-280.

39 Vanderstocken, G, Marrow, J P, Allwood, M A, et al. Disruption of physiological rhythms persist following cessation of cigarette smoke exposure in mice. Front Physiol. 2020; 11: 501383.

40 Bruijnzeel AW, Qi X, Guzhva LV, et al. Behavioral characterization of the effects of cannabis smoke and anandamide in rats. PLOS ONE 2016; 11: e0153327.

41 John G, Kohse K, Orasche J, et al. The composition of cigarette smoke determines inflammatory cell recruitment to the lung in COPD mouse models. Clin Sci (Lond)) 2014; 126: 207-221.

42 Dvorkin-Gheva A, Vanderstocken G, Yildirim, AÖ, et al. Total particulate matter concentration skews cigarette smoke's gene expression profile. ERJ open Res 2016; 2: 00029-2016.

43 Laniado-Laborin R. Smoking and chronic obstructive pulmonary disease (COPD). Parallel epidemics of the 21st century. Int J Environ Res Public Health 2009; 6: 209-224.

44 Barnes PJ. The cytokine network in chronic obstructive pulmonary disease. Am J Respir Cell Mol Biol 2009; 41: 631-638.

45 da Silva CO, Gicquel T, Daniel Y, et al. Alteration of immunophenotype of human macrophages and monocytes after exposure to cigarette smoke. Sci Rep 2020; 10: 12796.

46 Maloney JP, Gao L. Proinflammatory cytokines increase vascular endothelial growth factor expression in alveolar epithelial cells. Mediators Inflamm 2015; 2015: 387842. 